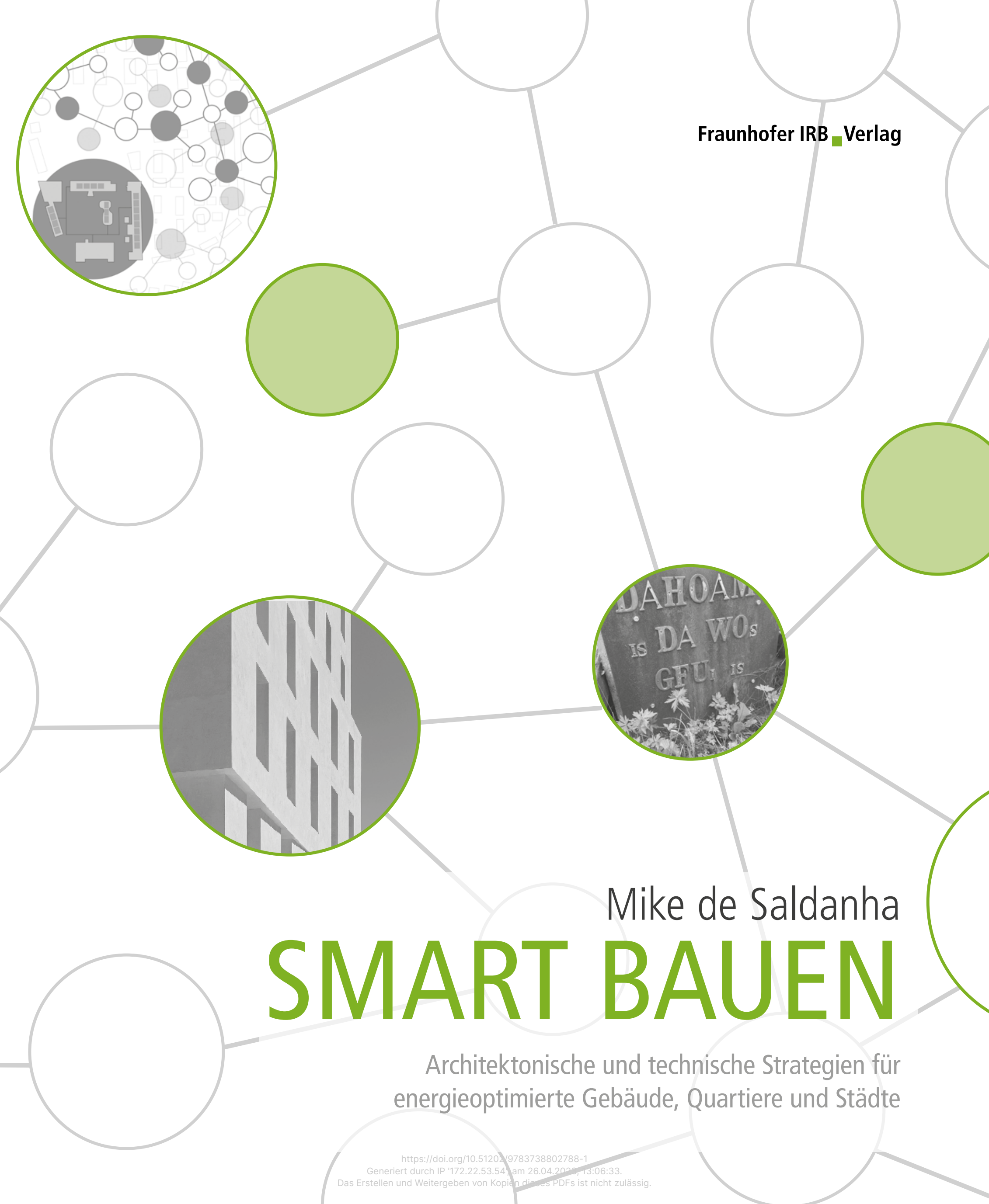


SMART BAUEN

- Fraunhofer IRB Verlag 
für

Christiane, Fabio, Emilio

gewidmet

Gerhard Hausladen 


\section{SMART BAUEN}

Architektonische und technische Strategien für energieoptimierte Gebäude, Quartiere und Städte

Prof. Dr. Mike de Saldanha 
Prof. Kristian Kaffenberger_Architekturbüro, Dilshofen

Dill Kahn_Atelier.Bim, Riedstadt

Benjamin Hossbach_[phase eins]., Berlin

Prof. Florian Nagler_Architekten GmbH, München

Johanna Uhland_Uhland GmbH, Darmstadt

Sibylle Bornefeld_Sauerbruch Hutton, Berlin

Martin Rauch_Lehm Ton Erde Baukunst, Schlins

Roger Boltshauser_Architekten, Zürich

Georg Bechter_Architektur + Design, Hittisau

Arnold Wild_Architekt, Konstanz

Dr. Michael Schäfer_ehemaliger Deutscher Botschafter in China

Özlem Gün_h_da, Hochschule Darmstadt

Prof. Dietmar Eberle_Baumschlager Eberle Architekten, Lustenau

Prof. Alexander Reichel_Architekten, Kassel

Karlheinz Beer_Büro für Architektur und Stadtplanung, München
Simon Speigner_sps, Thalgau

Prof. Hermann Kaufmann_HK Architekten, schwarzach

Peter Haimerl_Peter Haimerl.Architektur, München

Prof. Anke Mensing, Andreas Sedler_opus Architekten, Darmstadt

Frank Steinbacher_Steinbacher-Consult, Neusäß

Dr. Daniel Teichmann_Hydrogenious LOHC Technologies GmbH, Erlangen

Tobias Hoffmann_Vonovia AG, Bochum

Gerrit Ellerwald_Ampeers Energy GmbH, München

Dr. Karsten Schmidt_Ampeers Energy GmbH, München

Dr. Marc Weinstock_DSK-BIG, Kronshagen

Prof. Dr. Frank Bomarius_Fraunhofer IESE, Kaiserslautern

Jürgen Jost_ Jost Energy $A G$, Grünwald

Vinzenz Singer_Singer IC, Bayreuth

Rolf Disch_SolarArchitektur, Freiburg

Peter Mussaeus_PwC Legal, Frankfurt a. M. 
Mike de Saldanha

Autor

Sabine Uhland

Konzept, Layout, Grafiken, Fotos, Redaktion

Sigune Meister

Idee, Thematik, Lektorat 
Seit Anbeginn des energiesparenden Bauens haben sich verschiedene Strategien und Strömungen herausgebildet - angefangen bei einem auf aktive und passive Sonnenenergie gestützten Ansatz in den 1970er- und 1980erJahren, gefolgt von einer auf absolutes Einsparen basierten Strategie beim Passivhaus. Eine weitere Bandbreite ergibt sich aktuell im Technisierungsgrad, von absoluten LowtechGebäuden bis hin zu Hightech-Strategien, die auf die Potenziale der Digitalisierung und der künstlichen Intelligenz setzen.

Der Autor favorisiert keinen dieser Ansätze als alleiniges Allheilmittel, vielmehr gilt es, für die jeweilige Bauaufgabe und örtliche Situation aus den gegebenen Möglichkeiten ein ganzheitliches Konzept zu entwickeln, das den Nutzeranforderungen weitreichend entspricht. Deshalb besteht der Ansatz des vorliegenden Buches darin, eine Vielzahl von architektonischen, technischen, energetischen und raumklimatischen Strategien im Kontext mit ihren jeweiligen Protagonisten vorzustellen. Um die vielschichtige Thematik in der Linearität eines Buches abbilden zu können, wurde dieses in die Hauptkapitel »Mensch«, »Raum«, »Hülle«, »Haus», "Stadt» und »Land» untergliedert.

Mensch Die Aspekte der Behaglichkeit werden, gegliedert nach den Sinnen, thematisiert und dabei die wichtigsten planungsrelevanten Parameter zusammengestellt und erläutert. Die Herausforderungen und mögliche Strategien einer kreativen Konzeptentwicklung und
Planung werden diskutiert. Ein Exkurs führt in die Themengebiete »BIM « und »Energie im Architekturwettbewerb» ein.

Raum Die Zusammenhänge zwischen Lüftung und Raumkonditionierung werden beschrieben und diesbezügliche technische Systeme und Konzepte sowie ihre jeweiligen Einsatzbereiche aufgezeigt. Darüber hinaus werden die künstliche Beleuchtung und Tageslichtnutzung sowie die digitale Steuerung von technischen Systemen im Raum thematisiert.

Hülle Einer Zusammenstellung physikalischer Vorgänge an Fassaden folgen verschiedene Strategien für Dämmung und Sonnenschutz sowie mögliche Fassadenkonzepte. Es werden aktuelle Konstruktionsprinzipien im Detail gezeigt und innovative Materialien vorgestellt.

Haus Neben verschiedenen Energieerzeugungskonzepten für Gebäude werden raumklimatische Strategien für Wohn-, Verwaltungs-, Unterrichts- und Versammlungsgebäude aufgezeigt. Planungshinweise für die jeweiligen Nutzungen ergänzen die Erläuterungen.

Stadt In diesem Kapitel wird auf den aktuellen Trend der energetischen Betrachtung über das einzelne Gebäude hinaus, hin zu einem übergeordneten Konzeptansatz, der mehrere Gebäude, ein Quartier oder ganze Stadtteile einbezieht, eingegangen. Ein besonderer Schwerpunkt liegt dabei auf der Sektorenkopplung. 
Land Sowohl die ökonomischen als auch ökologischen Potenziale im Kontext der Energiewende werden aufgezeigt. Ein Überblick über aktuelle Gesetze sowie regulatorische Rahmenbedingungen vervollständigt das Werk.

Ausblick Der Autor wagt einen Blick in die Zukunft des energieoptimierten Bauens auf die Jahre 2030, 2040 und 2050.

Um die komplexen Themen anschaulich und praxisnah zu vermitteln, werden in allen Kapiteln Projektbeispiele vorgestellt und durch ein Statement des jeweiligen Planers ergänzt. Damit wird ein direkter Praxisbezug hergestellt. Die Betrachtung der ökonomischen Aspekte und Kosten sowie konkrete Planungsstrategien runden die unterschiedlichen Themenbereiche jeweils $a b$.

Das Buch wendet sich an Architekten, für die Behaglichkeit und niedriger Energieverbrauch wichtige Ziele ihrer Arbeit sind, und an Ingenieure, die eine Optimierung der Gebäudestruktur und Fassade erreichen möchten. Für Studierende führt es die Disziplinen »Entwurf», »Konstruktion«, »Bauphysik» und »Gebäudetechnik« ganzheitlich zusammen, sodass ein interdisziplinärer Blickwinkel bereits in der Ausbildung gegeben ist. Bauherren, Investoren und allen am Bauen Interessierten vermittelt es die Wissensgrundlage, um Konzepte, Entwürfe und Gebäude im Kontext der Energiewende kompetent beurteilen zu können und damit bessere Entscheidungen zu treffen.
Entsprechend der Vielschichtigkeit des Themas wurde das Buch im Dialog entwickelt. Für die fachliche Detaillierung haben zahlreiche Experten ihr Wissen einfließen lassen und viele Planer und Akteure ihre Praxiserfahrungen geteilt. Für den kreativen Input sei allen Beteiligten gedankt. Meiner Lektorin, Sigune Meister vom Fraunhofer IRB Verlag, gilt mein besonderer Dank dafür, die Thematik "Smart bauen« zur richtigen Zeit angestoßen und das Thema durch kritischen Dialog geschärft zu haben. Sabine Uhland danke ich für die innovative Konzeption des Buches, die frische grafische Umsetzung und die inspirierende, kreative Zusammenarbeit über drei Jahre hinweg.

Gewidmet ist dieses Werk Gerhard Hausladen, mit dem mich ein jahrzehntelanger gemeinsamer Weg beim innovativen Bauen verbindet und der mir ermöglicht hat, auf diesem Gebiet zu arbeiten und zu forschen. Ich verdanke dieser Inspiration meine berufliche Laufbahn und viele wertvolle Erfahrungen und Erkenntnisse, die in das vorliegende Buch eingeflossen sind.

Ich wünsche allen Leserinnen und Lesern viel Spaß und interessante Entdeckungen bei der Reise durch die Welt der Sinne, der Physik, der Architektur, der Technik und des Designs - der Welt des smarten Bauens.

München, Darmstadt, Port Adriano im April 2021

Mike de Saldanha 


\section{EINBLICK}

\section{INHALT}

I Zeitreise - Konzepte von früher bis heute

\section{MENSCH}

I Ganzheitliche Behaglichkeit

I Visuelle Behaglichkeit

I Akustische Behaglichkeit

I Thermische Behaglichkeit

I Olfaktorische Behaglichkeit

I Kreativität und Planung

I Building Information Modeling (BIM) 30

I Energie im Wettbewerb

I Naturhotel Tannerhof, Bayrischzell

\section{RAUM}

I Raumkonditionierung

I Natürliche Lüftung

I Mechanische Lüftung

I Raumkonditionierungssysteme

I Gebäudesteuerung und Smart Home 56

| »The Living - Microenergieapartment

I Speichermasse

I Technikintegration

| Tageslicht

9

34
$7 \quad$ I Kunstlicht

I Ökonomie der Raumkonditionierung 68

I Totenstube, Vrin

70

36

\section{6}

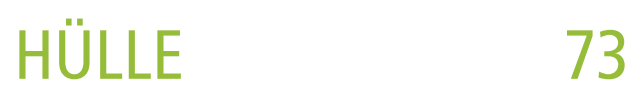

| Fassade als Schnittstelle 74

| Fassadenkonzepte 78

| Bauphysik der Hülle 84

I Wärmeschutz 86

| Anwendung von Dämmstoffen 90

I Glas 92

| Sonnenschutz 94

I Fassadenkonstruktionen 98

I Mehrschichtige Fassaden 100

I Monolithische Fassaden 102

| Holzkonstruktionen 104

I Holzbau 106

| Lehmbau 114

| Strohballenbau 118

| Bauen mit Bambus 120

| Begrünte Fassade 122

| Gebäudeintegrierte Photovoltaik 124

| Hybride Energiefassade 132

I Ökonomie der Gebäudehülle 136

I »Haus 2226« in Lustenau, Österreich 


\section{HAUS}

I Standortfaktoren

| Wärme- und

Kälteerzeugungssysteme

I Energiekonzepte für Gebäude

| Technikflächen

I Wohngebäude

| Gemischtgenutzte Gebäude

| Bürogebäude

I Unterrichtsgebäude

| Versammlungsgebäude

I Ökonomie der Energietechnik

I Wohn- und Bürohaus, Darmstadt
143

144

LAND

| Energiewende im Baubereich

I Digitalisierung

146 | 50 Jahre Energiegesetze in

150 Deutschland

154 I Energieeinsparverordnung (EnEV)

156

158 2016

226

I EnEV-Nachweis Wohngebäude

228

160 | EnEV-Nachweis Nichtwohngebäude 230

162 | Erneuerbare-Energien-Wärmegesetz 233

164 | Mieterstrommodell

234

166 | Kundenanlage

236

172

I Gebäudeenergiegesetz (GEG)

\section{STADT}

I Potenziale von Quartieren

| Mobilität

I Sortimo Innovationspark, Zusmarshausen

| Energiekonzepte für das Quartier

I Energiespeicherung

I Wasserstoff-Research-Center, Vonovia AG

I Sektorgekoppelte Quartiere

I Musterquartiere

I Ökonomie von Quartieren

I Brucklyn - "place to be« in Erlangen

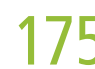

176
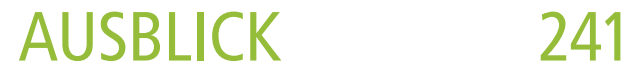

182

I 2020-2030 Die digitale Dekade

242

| 2030-2040 Die Green-Tech-Phase 244

| 2040-2050 Das interplanetare

186 Zeitalter

248

188

192

200

ANHANG

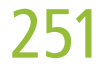

200

I Lebenswege und Stationen

252

202

I Literatur, Normen und Gesetze

254

206

I Fotografen und Bildnachweis

284

210

| Stichwortverzeichnis

290

I Impulse und Unterstützung

296

212

I Impressum 